\title{
Brain Surface Conformal Parameterization with Algebraic Functions
}

\author{
Yalin Wang ${ }^{1,2}$, Xianfeng Gu ${ }^{3}$, Tony F. Chan ${ }^{1}$, Paul M. Thompson ${ }^{2}$, \\ and Shing-Tung $\mathrm{Yau}^{4}$ \\ 1 Mathematics Department, UCLA, Los Angeles, CA 90095, USA \\ ${ }^{2}$ Lab. of Neuro Imaging, UCLA School of Medicine, Los Angeles, CA 90095, USA \\ ${ }^{3}$ Comp. Sci. Department, SUNY at Stony Brook, Stony Brook, NY 11794, USA \\ ${ }^{4}$ Department of Mathematics, Harvard University, Cambridge, MA 02138, USA \\ ylwang@math.ucla.edu
}

\begin{abstract}
In medical imaging, parameterized 3D surface models are of great interest for anatomical modeling and visualization, statistical comparisons of anatomy, and surface-based registration and signal processing. Here we introduce a parameterization method based on algebraic functions. By solving the Yamabe equation with the Ricci flow method, we can conformally map a brain surface to a multi-hole disk. The resulting parameterizations do not have any singularities and are intrinsic and stable. To illustrate the technique, we computed parameterizations of several types of anatomical surfaces in MRI scans of the brain, including the hippocampi and the cerebral cortices with various landmark curves labeled. For the cerebral cortical surfaces, we show the parameterization results are consistent with selected landmark curves and can be matched to each other using constrained harmonic maps. Unlike previous planar conformal parameterization methods, our algorithm does not introduce any singularity points. It also offers a method to explicitly match landmark curves between anatomical surfaces such as the cortex, and to compute conformal invariants for statistical comparisons of anatomy.
\end{abstract}

\section{Introduction}

Surface-based modeling is valuable in brain imaging to help analyze anatomical shape, to statistically combine or compare 3D anatomical models across subjects, and to map and compare functional imaging parameters localized on anatomical surfaces. Parameterization of these surface models involves computing a smooth (differentiable) one-to-one mapping of regular 2D coordinate grids onto the $3 \mathrm{D}$ surfaces, so that numerical quantities can be computed easily from the resulting models [1]. The mesh-based work contrasts with implicit methods, which typically define a surface as the level set of a higher-dimensional function [2]. Relative to level set methods, surface meshes can allow regular $2 \mathrm{D}$ grids to be imposed on complex structures, transforming a difficult $3 \mathrm{D}$ problem into a $2 \mathrm{D}$ planar problem, with simpler data structures, discretization schemes, and rapid data access and navigation. Here we present a new method to parameterize brain 
surfaces based on algebraic functions. We find a planar conformal parameterization without any singularities by solving the Yamabe equation with the Ricci flow method. This method can compute conformal invariants of brain surfaces which can be used to compare and classify brain surface structures. Compared with previous brain conformal parametrization work 34], the parameterization provided by our algorithm does not have any zero points so there is less area distortion. By solving a harmonic map in the parameter domain, our algorithm provides smooth correspondence fields for matching of different brain surfaces while explicitly matching labeled sets of landmark curves.

\subsection{Previous Work}

Brain surface parameterization has been studied intensively. Schwartz et al. [5], and Timsari and Leahy [6] computed quasi-isometric flat maps of the cerebral cortex. Drury et al. [7] presented a multiresolution method for flattening the cerebral cortex. Hurdal and Stephenson [8] report a discrete mapping approach that uses circle packings to produce "flattened" images of cortical surfaces on the sphere, the Euclidean plane, and the hyperbolic plane. The obtained maps are quasi-conformal approximations of classical conformal maps. Haker et al. 9] implement a finite element approximation for parameterizing brain surfaces via conformal mappings. They select a point on the cortex to map to the north pole of the Riemann sphere and conformally mapped the rest of the cortical surface to the complex plane by stereographic projection of the Riemann sphere to the complex plane. Gu et al. 10] propose a method to find a unique conformal mapping between any two genus zero manifolds by minimizing the harmonic energy of the map. They demonstrate this method by conformally mapping a cortical surface to a sphere. Ju et al. [11] present a least squares conformal mapping method for cortical surface flattening. Joshi et al. [12] propose a scheme to parameterize the surface of the cerebral cortex by minimizing an energy functional in the $p^{\text {th }}$ norm. Wang et al. 34 have used holomorphic 1-forms to parameterize anatomical surfaces with complex (possibly branching) topology. Recently, Ju et al. [13] reported the results of a quantitative comparison of FreeSurfer [14], CirclePack, and least squares conformal mapping (LSCM) with respect to geometric distortion and computational speed. They found that FreeSurfer performs best with respect to a global measurement of metric distortion, whereas LSCM performs best with respect to angular distortion and best in all but one case with a local measurement of metric distortion. Among the three approaches, FreeSurfer provides a more homogeneous distribution of metric distortion across the whole cortex than CirclePack and LSCM. LSCM is the most computationally efficient algorithm for generating spherical maps, while CirclePack is extremely fast for generating planar maps from patches.

\subsection{Theoretical Background and Definitions}

Given a multi-hole genus zero punctured surface, it is conformally equivalent to a special type of algebraic function,

$$
\omega^{2}=\left(z-z_{0}\right)\left(z-z_{1}\right)\left(z-z_{2}\right) \ldots\left(z-z_{2 g+1}\right),
$$


where $z_{0}, z_{1}, \ldots, z_{2 g+1} \in \mathbb{R}^{+}$are $2 g+2$ real numbers [15]. On the other hand, an algebraic function is conformally equivalent to a multi-hole punctured disk. Thus, for any genus zero surface with punctures, it can be shown that there exists a multi-hole punctured disk and a conformal map between the surface and the punctured disk. The disk is not unique, but any two such kind of punctured disks can be mapped to each other by a Möbius transformation $\tau$ of the form $\tau=\frac{a z+b}{c z+d}, a, b, c, d \in \mathbb{R}, z \in \mathbb{C}, a d-b c=1$.

The challenge of computing conformal maps between a punctured sphere to a multi-hole punctured disk lies in the fact that the range surface is underdetermined. Although it is clear that the boundaries of the domain surface will be mapped to circles on the plane, it is totally unknown what the centers and radii of these circles should be. These parameters need to be estimated during the computation process.

In the following, we formulate the conformal mapping problem in rigorous terms. Suppose $M$ is a genus zero surface, with $n+1$ holes, $N$ is a $n$-hole punctured disk, $\phi: M \rightarrow N$ is a conformal map between them, $g$ is the metric tensor on $M$, usually $g$ is induced by the ambient Euclidean metric of $\mathbb{R}^{3}$.

From the theory of complex geometry, the problem of finding a conformal map, $\phi: M \rightarrow N$, is equivalent to finding a function $u: M \rightarrow \mathbb{R}$, such that $\tilde{g}=e^{2 u} g$ is the metric on $M$ induced by $\phi$, which satisfies the Yamabe equation [16].

$$
\left\{\begin{aligned}
\tilde{K} & =0 \\
\Delta u-K+e^{2 u} \tilde{K} & =0 \\
\left.k_{\tilde{g}}\right|_{\partial M} & =\text { const }
\end{aligned}\right.
$$

where $\tilde{K}$ is the target Gaussian curvature and $K$ is the original Gaussian curvature. Basically, the domain surface $M$ is conformally deformed such that the Gaussian curvature of the interior points is zero everywhere and the boundaries become circles.

We can solve Equation 2 with the Ricci flow [17,

$$
\frac{d u(t)}{d t}=\tilde{K}-K(t)
$$

where $\tilde{K}$ is the target Gaussian curvature and $K$ is the Gaussian curvature at time t. We study a conformal deformation that deforms the surface accordingly until the maximal Gaussian curvature error $\max _{t}|\tilde{K}-K(t)|$ falls below a given threshold.

\section{Algorithms}

In section 2.1 we explain the algorithm details to conformally map an open boundary genus zero surface to a multi-hole punctured disk. In section 2.2. we explain how to match two open boundary genus zero surfaces via their conformal parameterizations using multi-hole punctured disks. 


\subsection{Conformal Mapping to a Multi-hole Punctured Disk}

The algorithm is equivalent solving Equation 2 that describes a conformal deformation. We use the Ricci flow method [17 to solve this equation. We adopted the same way to compute Gaussian Curvature in [18.

Algorithm 1. Conformal Mapping to a Multi-hole Punctured Disk Input: mesh $M$, step length $\epsilon$, energy difference threshold $\delta K$; Output: $\boldsymbol{h}: M \rightarrow D$. Here $D \in R^{2}$, and $D$ is a multi-hole disk.

1. Computing initial radii $\gamma_{i}$ for each vertex, and angle $\phi_{i j}$ for each edge $e_{i j}$, such that $l_{i j}=\gamma_{i}^{2}+\gamma_{j}^{2}-2 \gamma_{i} \gamma_{j} \cos \phi_{i j}$.

2. Compute boundary loops, denoted as $\Gamma_{0}, \Gamma_{1}, \ldots, \Gamma_{n}$. The $\Gamma_{0}$ is the exterior boundary.

3. Set target Gaussian curvature of each interior vertex to be zero, $\tilde{K}_{i}=0$.

4. For any vertex on $v_{k} \in \Gamma_{0}$, set its target Gaussian curvature to $\tilde{K}_{k}=\frac{2 \pi}{\left|\Gamma_{i}\right|}$, where $\left|\Gamma_{0}\right|$ denotes the number of vertices in $\Gamma_{0}$.

5. For any vertex on $v_{k} \in \Gamma_{i}, i \neq 0$, set its target Gaussian curvature to $\tilde{K}_{k}=-\frac{2 \pi}{\left|\Gamma_{i}\right|}$, where $\left|\Gamma_{i}\right|$ denotes the number of vertices in $\Gamma_{i}$.

6. Update the vertex radii with the Ricci flow,

$$
\gamma_{i}(t+1)=\gamma_{i}(t)+\epsilon \times\left(\tilde{K}_{i}(t)-K_{i}(t)\right) \times \gamma_{i}(t) .
$$

7. Update the target Gaussian curvature for boundary vertices, suppose $v_{k} \in \Gamma_{i}$, suppose $e_{k-1, k}, e_{k, k+1} \in \Gamma_{i}$, then let $S_{i}=\Sigma_{e_{p q} \in \Gamma_{i}} l_{p q}$, then

$$
\tilde{K}_{k}=\frac{l_{k-1, k}+l_{k, k+1}}{2 S_{i}} \times C_{i}
$$

where $C_{i}$ is $C_{i}=\left\{\begin{array}{r}2 \pi, i=0 \\ -2 \pi, i \neq 0\end{array}\right.$.

8. Repeat step 6 and 7 until the maximal Gaussian curvature error, $\max _{i} \mid K_{i}-$ $\tilde{K}_{i} \mid$, is less than $\delta K$.

\subsection{Surface Matching with Punctured Disk Parameterization}

After the computation of conformal parameterizations for open boundary genus zero surfaces with a multiple-hole punctured disk, we can compute the direct correspondence of two surfaces by solving a constrained harmonic mapping problem [4]. Given two surfaces $S_{1}$ and $S_{2}$, their punctured disk parameterizations are $\tau_{1}: S_{1} \rightarrow R^{2}$ and $\tau_{2}: S_{2} \rightarrow R^{2}$, we want to compute a map, $\phi: S_{1} \rightarrow S_{2}$. Instead of directly computing of $\phi$, we can easily find a harmonic map between the parameter domains. We look for a harmonic map, $\tau: R^{2} \rightarrow R^{2}$, such that $\tau \circ \tau_{1}\left(S_{1}\right)=\tau_{2}\left(S_{2}\right), \tau \circ \tau_{1}\left(\partial S_{1}\right)=\tau_{2}\left(\partial S_{2}\right), \Delta \tau=0$. Then the map $\phi$ can be obtained by $\phi=\tau_{1} \circ \tau \circ \tau_{2}^{-1}$. Since $\tau$ is a harmonic map while $\tau_{1}$ and $\tau_{2}$ are conformal map, the resulting $\phi$ is a harmonic map. 


\section{$3 \quad$ Experimental Results}

We applied our algorithms to parameterize various anatomic surfaces extracted from 3D MRI scans of the brain. We tested our algorithm on a left hippocampal surface, a structure in the medial temporal lobe of the brain. The original surface is shown in Figure 1(a). We leave two holes on the front and back of the hippocampal surface, representing its anterior junction with the amygdala, and its posterior limit as it turns into the white matter of the fornix. It can be logically represented as an open boundary genus one surface, i.e., a cylinder (note that spherical harmonic representations would also be possible, if ends were closed [19]). Its conformal map to a 1-hole disk is illustrated in Figure1(d). For the two boundaries of the hippocampal surface, one boundary is mapped to the exterior circle and the other is mapped to the internal circle.

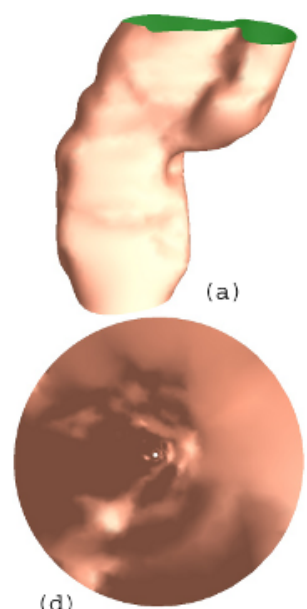

(d)

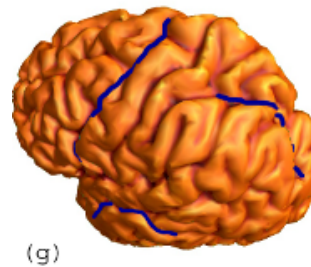

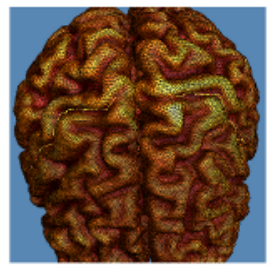

(b)

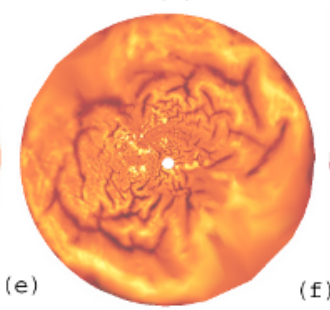

(e)

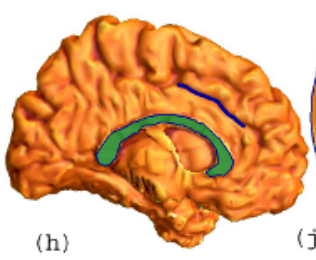

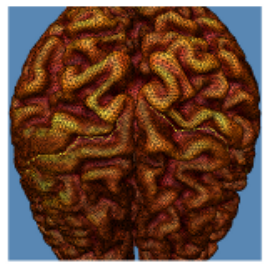

(c)

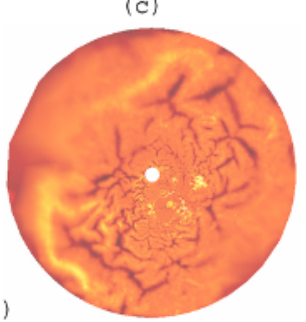

(f)

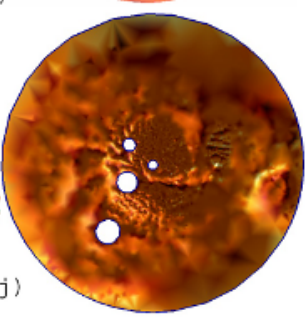

Fig. 1. Illustrates conformal maps from hippocampal and cortical surfaces to multi-hole disks. (a) shows the front view of a hippocampal surface and (d) shows its conformal map to a 1-hole disk. (b) and (c) are two cerebral cortical surfaces. Two central sulci are labeled as yellow curves on each of them. After cutting along the landmark curves, each of these two surfaces can be conformally mapped to a 1-hole disk ((e) and (f)). The radii of the inner circles are conformal invariants of two surfaces and can be used as shape index to compare and classify brain surfaces. (g)-(j) show a conformal map from a left hemisphere cortex with 5 labeled landmarks to a 4-hole disk. (g) and (h) show the front and back side of the surface. (j) shows its conformal map on a 4-hole disk. 
We also applied our algorithm to parameterize the surface of the cerebral cortex. The cerebral cortex and landmark data are the same ones used in [20]. We tested our algorithm with different landmark sets. Figure 1(b) and (c) show two cortical surfaces with two central sulci on two hemispheres. After we cut the cortical surface open along the two central sulci, the cortical surface is topologically equivalent to an open boundary genus one surface. Figure[1(e) and (f) show their conformal parameterizations in a 1-hole disk. One of the two landmark curves is mapped to the exterior circular boundary and the other is mapped to the inner circular boundary. The circle center positions and radii are two conformal invariants of the cortical surfaces studied. For example, the inner circle centers of (e) and (f) are in the origins but the radius of (e) is 0.48 while the inner circle radius of (f) is 0.53 . These parameters are surface conformal invariants and may be used as a shape index to classify and compare different cortical surfaces.

Figure 1(g) and (h) show a left hemisphere cortex with 5 landmarks labeled (thick lines show the precentral sulcus, the superior temporal sulcus, the intraparietal sulcus, the anterior segment of cingulate sulcus and the corpus callosum boundary at the midsagittal plane). After cutting along these landmark curves, the cortical surface becomes an open boundary genus-4 surface. Our algorithm conformally maps the surface to a 4-hole disk (Figure[1(j)). The boundary circle of the corpus callosum is mapped to the exterior circular disk boundary and other four landmark curves are mapped to the disk inner circle boundaries.

Figure 2 illustrate how our algorithm is used to match two left hemisphere cortical surfaces. As shown in Figure 2(a), (b), (d) and (e), we selected four major landmark curves on two different cortices, to illustrate the approach (thick lines show the precentral and postcentral sulci, the superior temporal sulcus, and the corpus callosum boundary at the midsagittal plane). By cutting the surface along these landmark curves, we obtain two genus-3 open-boundary surfaces. Figure 2(c) and (f) show their conformal map to a 3-hole disk. Because of the shape difference between two cortices, the centers and the radii of inner circles are different. By computing a constrained harmonic map from (f) to (c), we have a new parameterization (Figure $2(\mathrm{~g})$ ) of the cortex in the second row $((\mathrm{d})$ and (e)). The inner circle centers and radii of the new parameterization are identical to the parameterization in (c). With the new 3-hole disk as the canonical space, we can easily compute a direct surface correspondence between two surfaces (a) and (d). Because the inner circles and exterior circle are identical for two parameterizations, the landmark curves lying in the surface are exactly matched to each other. Figure 2 (h)-(m) illustrate the direct surface correspondence by morphing between these two cortical surfaces. Figure $2(\mathrm{~h})$ and $(\mathrm{m})$ and surfaces (a) and (d) respectively, viewed from a different viewpoint. (j), (k) and (l) are the intermediate shapes when linearly interpolating the surface correspondence vector field between $(\mathrm{h})$ and $(\mathrm{m})$. We can see that although surface shape changes substantially from $(\mathrm{h})$ to $(\mathrm{m})$, the relative locations of the three landmarks remain the same. This verifies that our algorithm provides a method to perform surface matching, while explicitly matching sulcal curves or other landmarks lying in the surface. Note although some previous approaches [34 had the same motivation 


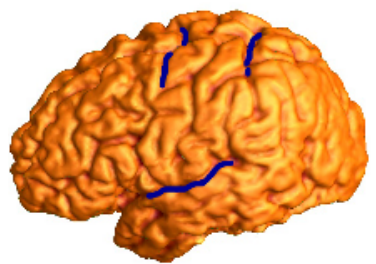

(a)

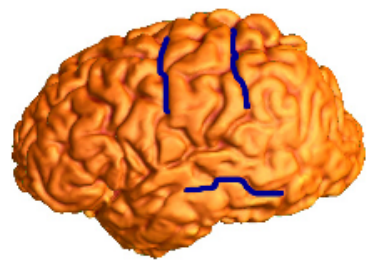

(d)
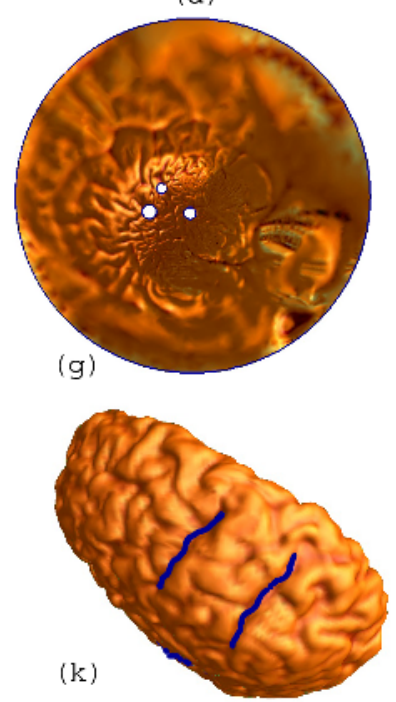

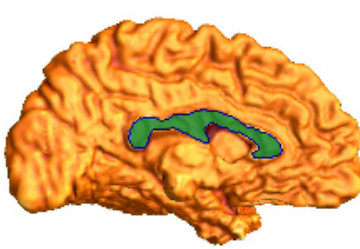

(b)

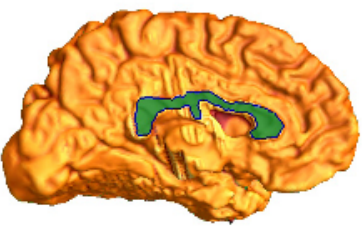

(e)
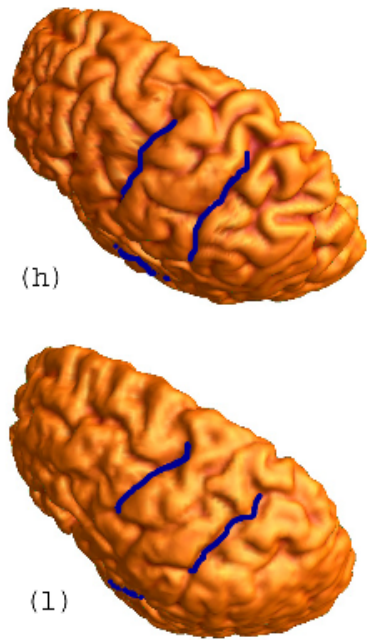
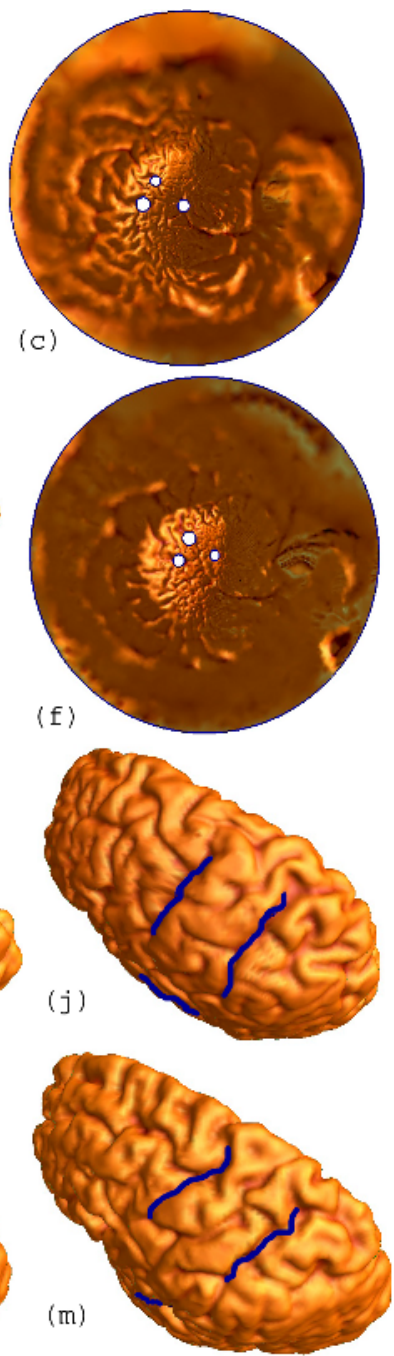

Fig. 2. Illustrates direct surface matching between two different cerebral cortical surfaces while explicitly matching landmark curves. (a)-(b) show a left cerebral cortex with four labeled landmarks and (c) shows its conformal map to a 3-hole disk. (d)-(e) show another left hemisphere model of the cerebral cortex with the same landmarks labeled and (f) shows its conformal map to a 3-hole disk. (g) is the parameterization of surface (d)-(e) after a constrained harmonic map from (f) to (c) is built. (h)-(m) show a morphing sequence from surface (a)-(b) to surface (d)-(e). (j)-(l) are the intermediate shapes when we linearly interpolate surface correspondence vector field between two surfaces $(\mathrm{h})$ and $(\mathrm{m})$. Although the cortical surface shape changes considerably, the relative positions of the selected landmark curves do not change. 
as ours, because their work introduced singularities at the so-called zero points, their surface matching results have some errors and inevitable distortions in the areas around the zero points. Our approach provides an improved method for global surface matching with exact landmark matching capability.

\section{Conclusion and Future Work}

In this paper, we present a brain surface conformal parameterization method based on algebraic functions. With the Ricci flow method, we solve the Yamabe equation to obtain a conformal deformation that conformally maps open boundary surfaces to multi-hole disk. We tested our algorithm on the hippocampus and surface models of the cerebral cortex. Used as a canonical space, the multi-hole disk conformal parameterization provides a brain surface matching approach that can exactly match landmark curves lying on the surfaces. Compared with other work which conformally maps brain surfaces to parallelograms, our algorithm offers some advantages because it does not introduce any singular points. Our future work will include algebraic function computation based on the multi-hole disk parameterization and empirical application of these Yamabe flow concepts to medical applications in computational anatomy.

\section{References}

1. Thompson, P.M., Giedd, J.N., Woods, R.P., MacDonald, D., Evans, A.C., Toga, A.W. Nature 404(6774) (2000) 190-193

2. Osher, S., Sethian, J.A. J. Comput. Phys. 79 (1988) 12-49

3. Wang, Y., Gu, X., Hayashi, K.M., Chan, T.F., Thompson, P.M., Yau, S.T. In: MICCAI. Volume II., Palm Springs, CA, USA (2005) 26-29

4. Wang, Y., Gu, X., Hayashi, K.M., Chan, T.F., Thompson, P.M., Yau, S.T. In: 10th IEEE ICCV. (2005) 1061-1066

5. Schwartz, E., Shaw, A., Wolfson, E. IEEE TPAMI 11(9) (1989) 1005-1008

6. Timsari, B., Leahy, R.M. In: Proceedings of SPIE, Medical Imaging, San Diego, CA (2000)

7. Drury, H.A., Van Essen, D.C., Anderson, C.H., Lee, C.W., Coogan, T.A., Lewis, J.W. J. Cognitive Neurosciences 8 (1996) 1-28

8. Hurdal, M.K., Stephenson, K. NeuroImage 23 (2004) S119-S128

9. Angenent, S., Haker, S., Tannenbaum, A., Kikinis, R. Med. Image Comput. Comput.-Assist. Intervention (1999) 271-278

10. Gu, X., Wang, Y., Chan, T.F., Thompson, P.M., Yau, S.T. IEEE TMI 23(8) (2004) 949-958

11. Ju, L., Stern, J., Rehm, K., Schaper, K., Hurdal, M.K., Rottenberg, D. In: IEEE ISBI, Arlington, VA, USA (2004) 77-80

12. Joshi, A.A., Leahy, R.M., Thompson, P.M., Shattuck, D.W. In: IEEE ISBI, Arlington, VA, USA (2004) 428-431

13. Ju, L., Hurdal, M.K., Stern, J., Rehm, K., Schaper, K., Rottenberg, D. NeuroImage 28(4) (2005) 869-880

14. Fischl, B., Sereno, M.I., Dale, A.M. NeuroImage 9 (1999) 179-194

15. Springer, G.: Introduction to Riemann Surfaces. AMS Chelsea (2000) 
16. Schoen, R., Yau, S.T.: Lectures on Differential Geometry. International Press (1994)

17. Hamilton, R.S.: The Ricci flow on surfaces. Contemp. Math. 71 (1988) 237-262

18. Wang, Y., Chiang, M.C., Thompson, P.M. In: Med. Image Comp. Comput.-Assist. Intervention, Proceedings, Part II. (2005) 666-674

19. Kelemen, A., Székely, G., Gerig, G. IEEE TMI 18(10) (1999) 828-839

20. Thompson, P.M., Lee, A., Dutton, R., Geaga, J., Hayashi, K., Eckert, M., Bellugi, U., Galaburda, A., Korenberg, J., Mills, D., Toga, A., Reiss, A. J. Neuroscience 25(16) (2005) 4146-4158 